\section{Digital dental photography. Part 9: post-image capture processing}

\author{
I. Ahmad ${ }^{1}$
}

IN BRIEF

- Editing an image causes deterioration in quality, is complicated, time consuming, onerous and frustrating.

- Ethically acceptable alterations include correcting exposure, orientation, laterally inverting and cropping an image.

- The most popular file formats to consider are RAW data, TIFF and JPEG.

- The most expedient and eco-friendly transfer of images is via the Internet.

Having successfully taken a digital image, the next step is deciding what to do with it. Should it be cropped, correctly orientated, manipulated, compressed, scaled, sharpened, archived (and if so, which file format is the most suitable), or even discarded? The premise of this part of our series is to answer these and other questions related to post-production of a digital image.

With regard to manipulation, it is important to remember that dental images are dentolegal documents. Therefore, manipulation should be kept to a minimum, ensuring that the original image is not altered to an extent that hides pathology or alters the clinical situation to camouflage what was present in the oral cavity. Current photo-editing software allows an image to be manipulated beyond recognition, and while this is acceptable for dramatic or artistic purposes, it is inappropriate for dental imagery. Altering exposure,

\section{FUNDAMENTALS OF DIGITAL DENTAL PHOTOGRAPHY}

1. Digital dental photography: an overview

2. Purposes and uses

3. Principles of digital photography

4. Choosing a camera and accessories

5. Lighting

6. Camera settings

7. Extra-oral set-ups

8. Intra-oral set-ups

9. Post-image capture processing

10. Printing, publishing and presentations

${ }^{1}$ General Dental Practitioner, The Ridgeway Dental Surgery, 173 The Ridgeway, North Harrow, Middlesex, HA2 7DF

Correspondence to: Irfan Ahmad

Email: iahmadbds@aol.com

www.IrfanAhmadTRDS.co.uk

\section{Refereed Paper}

Accepted 15 November 2008

DOI: 10.1038/sj.bdj.2009.763

${ }^{\circledR}$ British Dental Journal 2009; 207: 203-209 orientation or cropping extraneous parts is acceptable, and indeed desirable to visualise the clinical situation as it appeared at the time of taking the picture. Hence, this chapter will only cover manipulation that is deemed ethically acceptable for dentistry.

Another important point worth mentioning is that image quality is directly related to the degree of manipulation. The greater the manipulation, the poorer the image quality. Therefore, it is crucial to keep alterations to a minimum by ensuring that the original image capture was as perfect as possible regarding exposure, magnification, orientation and composition. Furthermore, photo-editing software is complicated, applications often requiring training and being very time intensive.

\section{INITIAL PROCESSING}

The physical transfer of images from the camera into computer-based software is by one of the following methods:

1. USB-2 cable connection

2. FireWire ${ }^{\circledast} 400$ or FireWire $800^{\circ}$

cable connections

3. Wireless connection.

The method of transfer depends on the camera and computer ports. A USB-2 cable is sufficiently fast for small files, but extremely slow for larger files. In these circumstances, FireWire 400 , or the faster FireWire $800^{\circ}$ cables are the ideal choice. The latest transfer mode is wireless connections, which eliminate cables but at present are relatively slow. In some instances a print may be immediately required, and in these circumstances the camera or its memory card can be directly connected via a USB cable, or inserted into an office printer, respectively.

It is debatable whether a Windows ${ }^{\mathrm{TM}}$ or Macintosh ${ }^{\mathrm{TM}}$ based computer is more appropriate for image management and manipulation. Windows-based PCs have the lion's share of the market, while Apple ${ }^{\circledast} \mathrm{Mac}^{\mathrm{TM}}$ machines are more eclectic. Previously, the Windows platform was relatively slow in handling graphics, being more suited for applications such as word processing, accounting and databases. However, the latest version, Windows Vista ${ }^{\mathrm{TM}}$, promises to rival the Apple OSX operating system Snow Leopard with regard to image management. There is no doubt that professional photographers, graphic designers and printings houses have a penchant for Apple Macs due to their superior capabilities for handling, manipulating and storing image files. For this reason, if budgets allow and one is taking large volumes of dental images, the Apple Mac is the ideal choice. On the other hand, for small volume documentation a Windows-based PC is adequate. Finally, compatibility was previously an issue between Windows and Macintosh platforms, however the newer versions of both operating systems allow free exchange of files, without the need for conversion or filters.

The first thing is to decide whether an image is useable or should be discarded. 
While image manipulation can correct many failings in technique, it cannot perform miracles. If an image is grossly underor over-exposed, it is probably prudent to delete it and start again. Also, images that do not reveal items (or details of items) that were sought should also have a similar demise. For example, if the purpose of the intended image was to show translucency or characterisations within a tooth but it is over-exposed, no amount of manipulation will reveal the missing translucency or characterisations. In a similar vein, if an image is out of focus or lacking detail in specific areas due to a small dynamic range, no software can be expected to put back or replace something that was lacking or absent in the beginning. Furthermore, excess manipulation is time consuming and causes severe deterioration in image quality. Therefore it may be expedient and easier to take another picture rather than labouring with software to achieve the impossible and ending up with a result that lacks quality and will ultimately be of little use.

If not already performed by camera settings, the first item for post-image production is to ensure the correct white balance for the prevailing lighting conditions at the time of exposure. Setting the white balance was discussed in Part $6^{1}$ and can be calculated automatically by camera electronics, manually inputted, or calibrated with an $18 \%$ grey card. If the last option was chosen, a single image or multiple images are selected in the chosen software (either proprietary or Adobe PhotoShop), and the grey balance settings recalled for calibrating the new images (Figs 1-2).

\section{Correcting orientation, exposure, laterally inverting and cropping}

A major difficulty with dental photography is framing the picture with correct orientation for ensuring extraneous items such as saliva ejectors, cheek retractors and cotton wool rolls are invisible. This was of course challenging for film photography, since little post-production was possible. However, with digital photography, changing orientation, cropping or altering the exposure (within limits) is a relatively simple task. The latter can either be performed in-camera, with camera specific software, or in photo-editing software such as Adobe ${ }^{\circledast}$ PhotoShop.

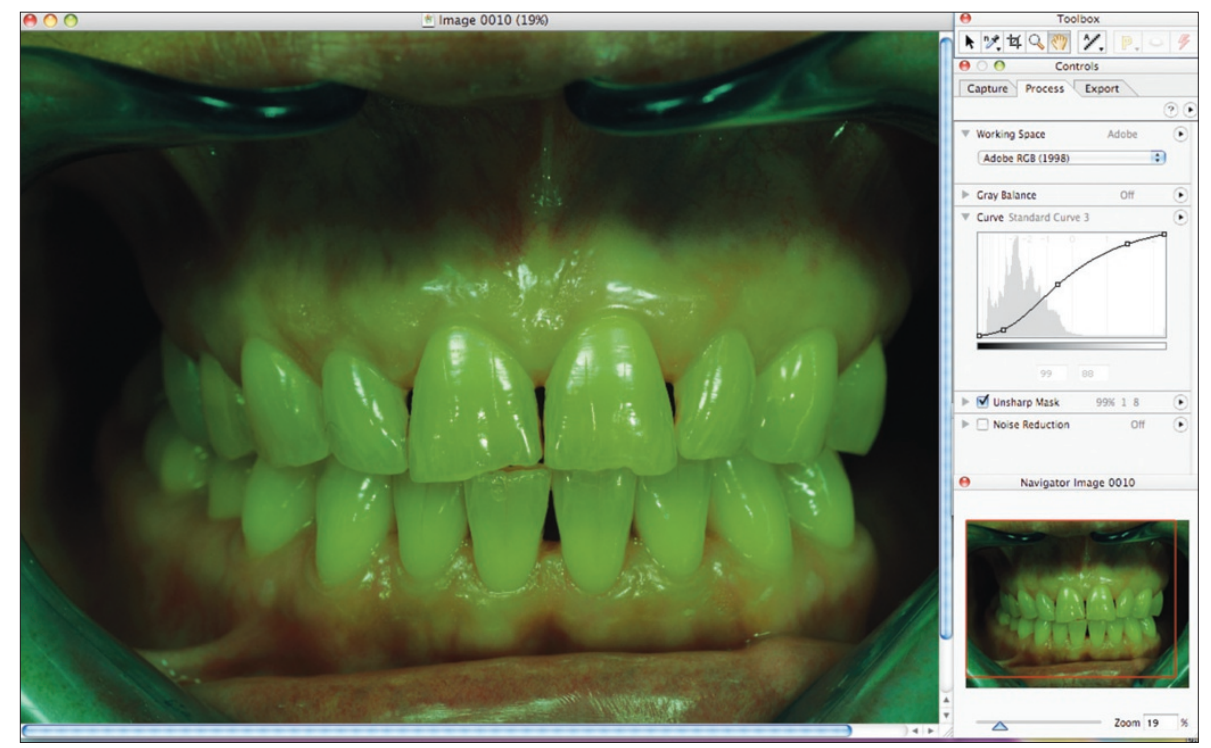

Fig. 1 The raw image from the camera without white balance calibration (notice 'Gray Balance Off' on menu tab)

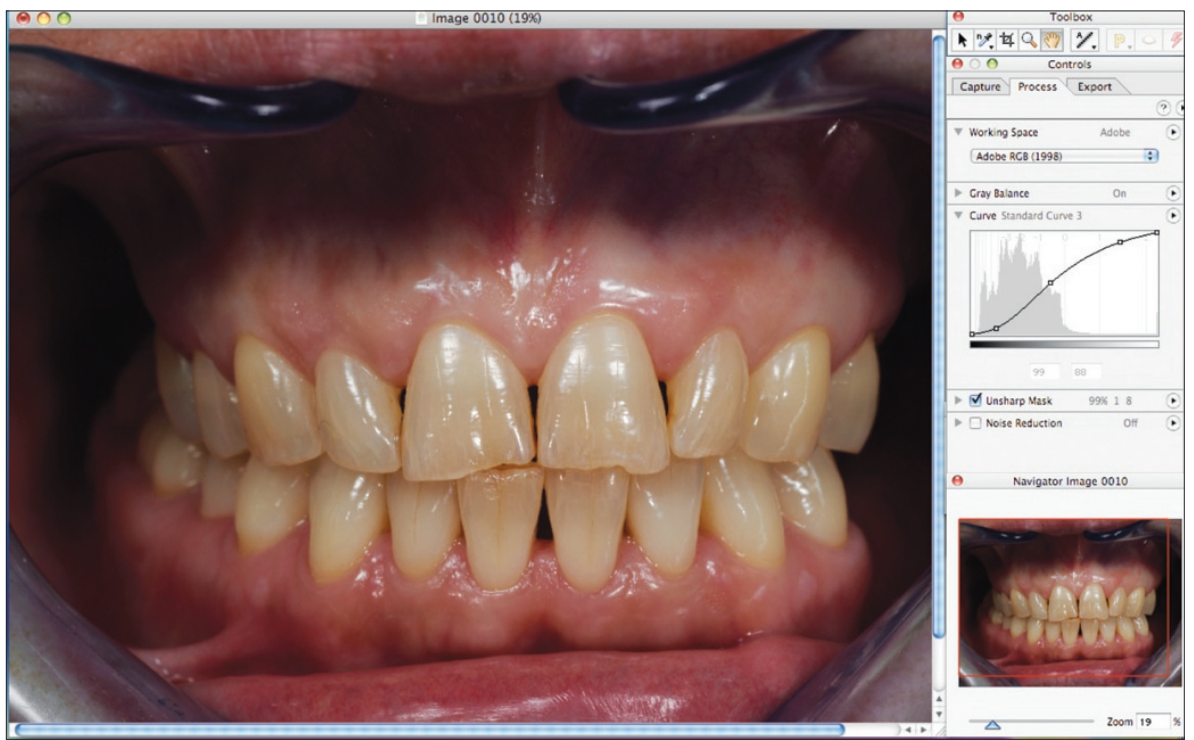

Fig. 2 The same image as Figure 1 with white balance corrected by recalling the grey card calibration (notice 'Gray Balance On' on menu tab)

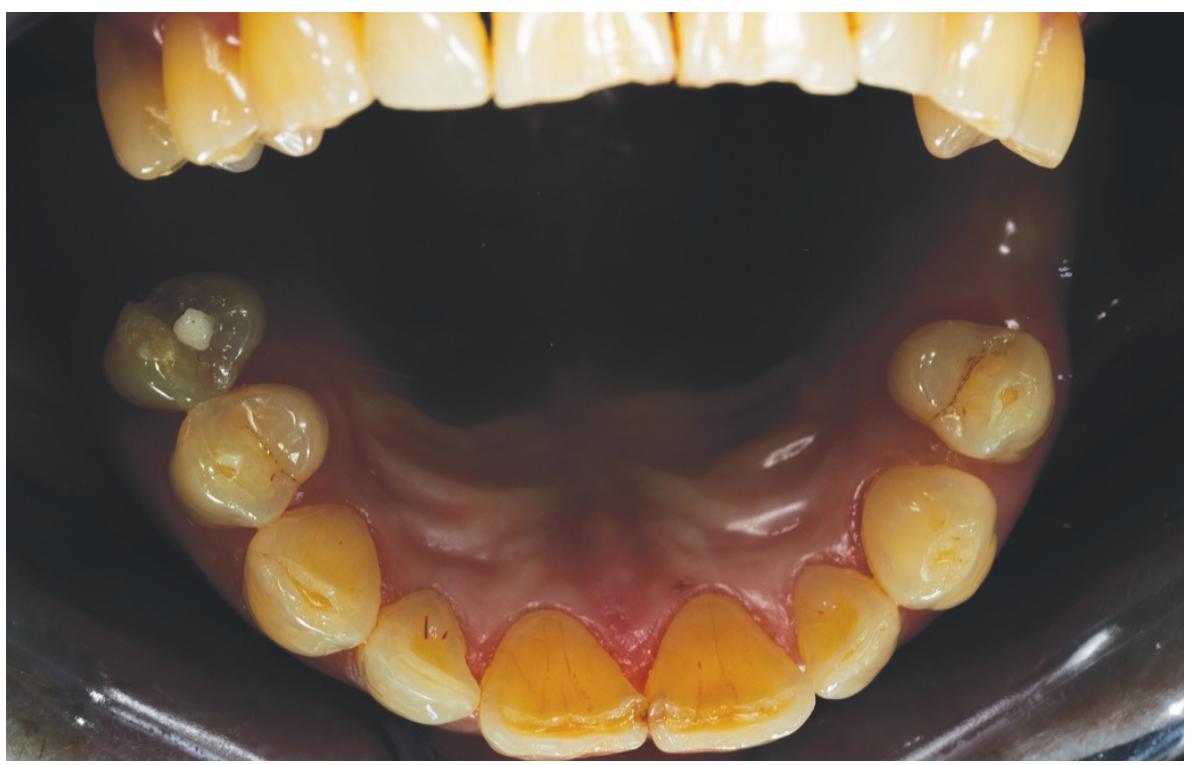

Fig. 3 Initial image from digital camera 
The order in which correcting exposure, orientating, laterally inverting or cropping is performed is irrelevant. Furthermore, every type of software has its own commands and methods for executing the above corrections. However, for illustration purposes, the image in Figure 3 was edited in Adobe ${ }^{\circledast}$ PhotoShop (Figs 4-7) and the final result is shown in Figure 8. Images which are taken using an intra-oral mirror are laterally inverted and require subsequent correction, while those without will not require the addition correction shown in Figure 7.

\section{Scaling}

Scaling or enlarging an image may often be necessary, for example after cropping or for concentrating on specific detail. Any scaling causes image deterioration and this is the major reason that high quality and quantity of pixels is essential for recording as much detail as possible at the outset. The mathematical enlargement of an image is termed interpolation. The resulting image quality after enlargement depends on the algorithms used for interpolation. PhotoShop offers a variety of algorithms for interpolation, including nearest neighbour, bi-linear, bicubic, fractals and reduction. For modest enlargements, nearest neighbour or bi-linear is adequate, but for larger scaling bicubic, fractals and reduction are better choices. However, scaling is not limitless. If the original image is enlarged excessively, pixelation will occur and the scaling process for enlarging specific details will obviously be futile. Therefore, marked enlargement to the extent where an image breaks down is pointless, and defaces the image beyond recognition.

Ideally, an image should require minimal or no sharpening and if blurring is pronounced, it is better to take another picture that is sharply focused. An important point to remember is that if sharpening is necessary it should always follow scaling, not precede it. If sharpening is performed before enlarging an image any artefacts such as contour fringes at the edge of objects will also be enlarged and become more apparent. Furthermore, excess sharpening introduces grain and noise, which may defeat the initial objective of sharpening the image.

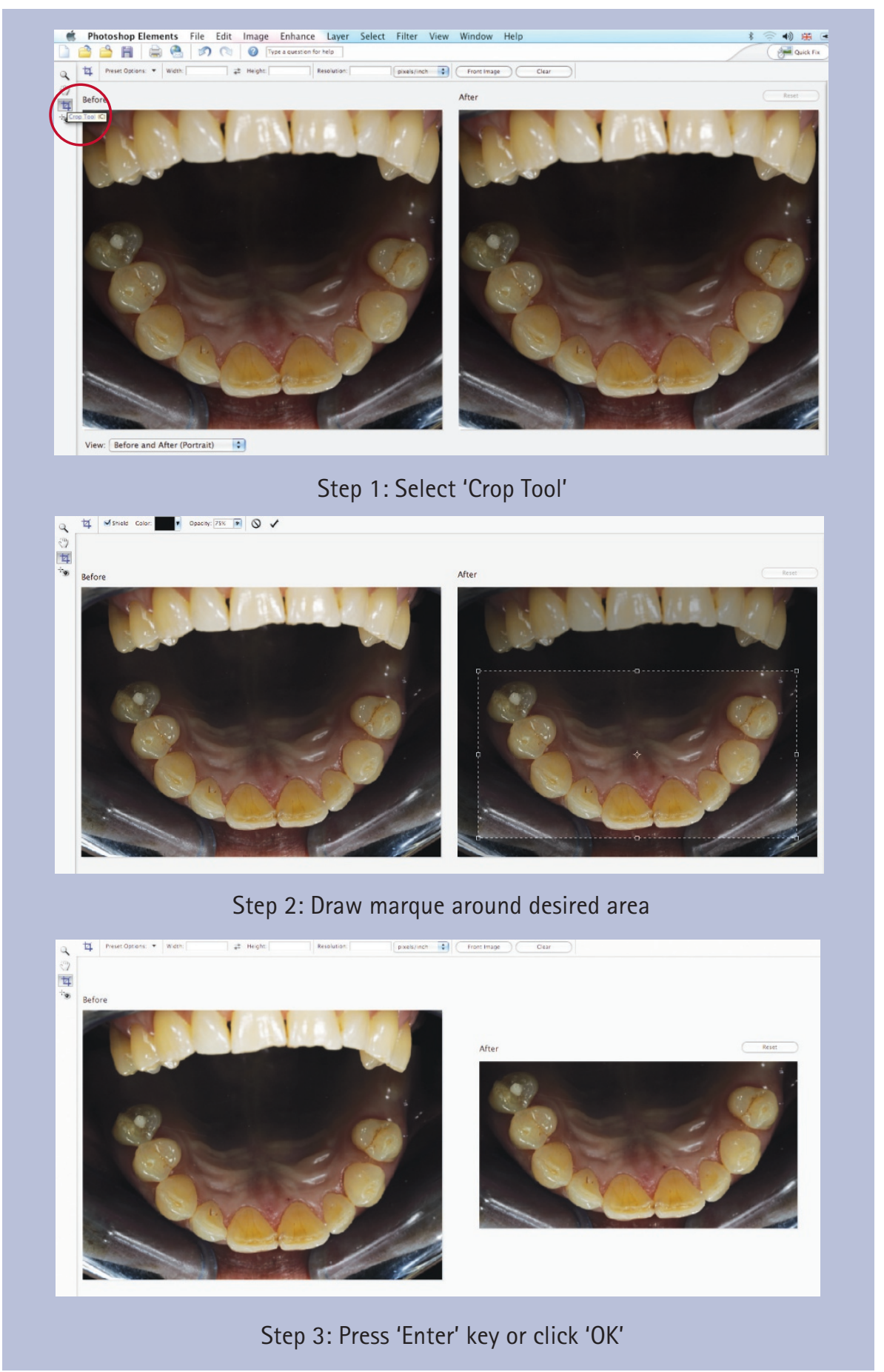

Fig. 4 Cropping: procedure for cropping an image

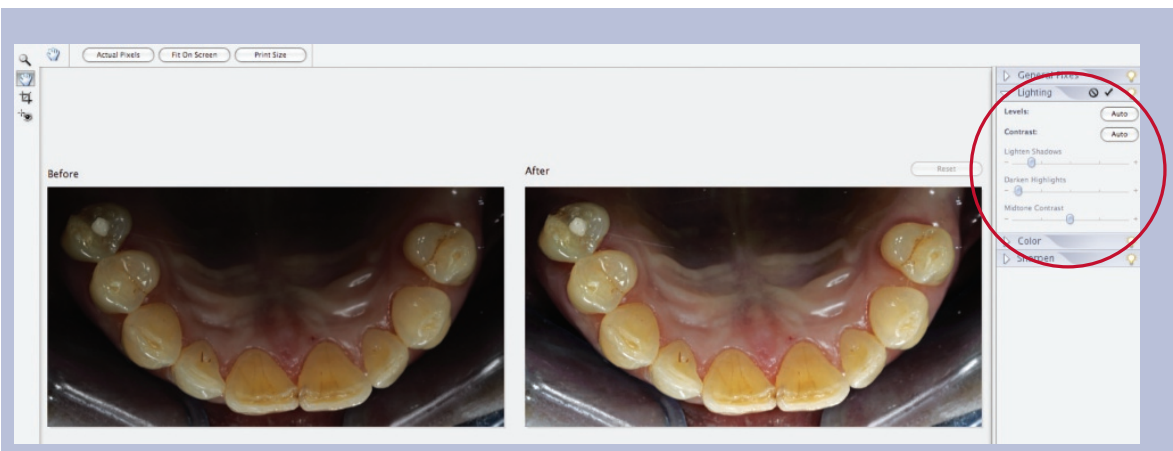

Adjust levels until desired exposure is obtained

Fig. 5 Exposure: procedure for correcting exposure 


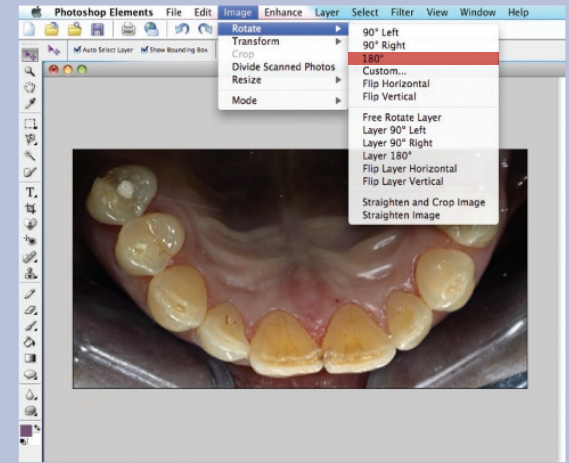

Step 1: Select 'Image: Rotate: $180^{\circ}$ from menu bar to upright image

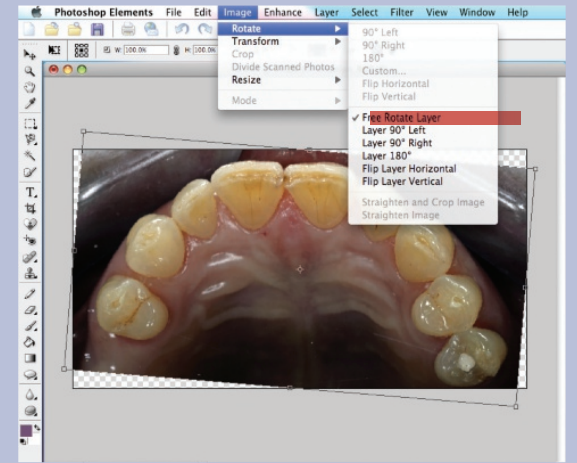

Step 2: Select 'Image: Rotate: Free Rotate Layer' to finely rotate image to horizontal

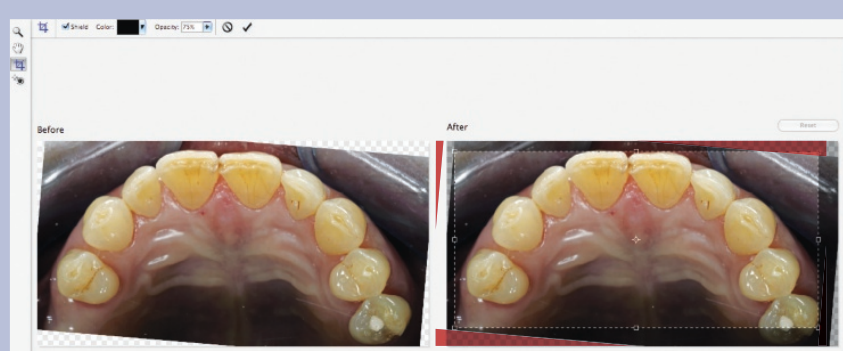

Step 3: Crop if necessary, to remove superfluous pats of the image

Fig. 6 Orientation: procedure for correcting orientation

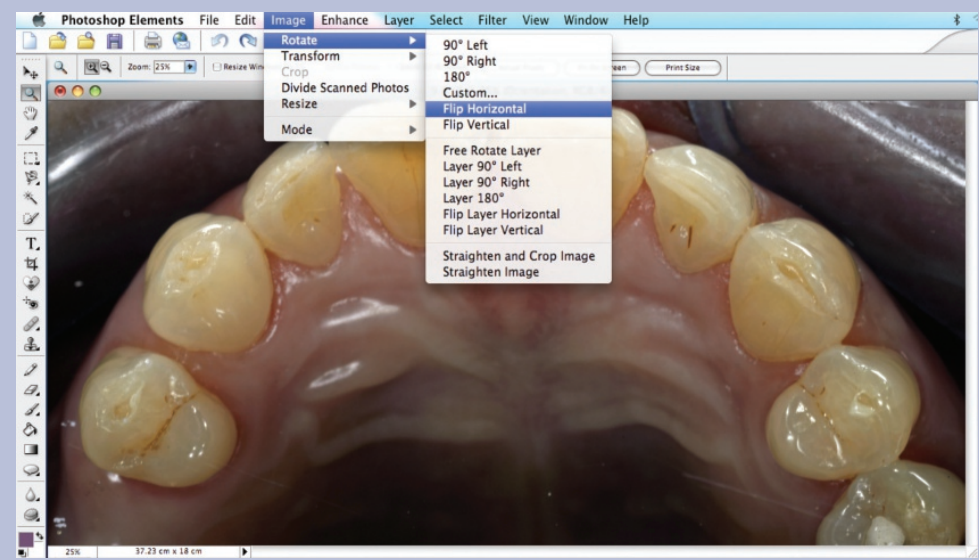

Select 'Image: Rotate: Flip Horizontal' from menu bar

Fig. 7 Procedure for laterally inverting the image

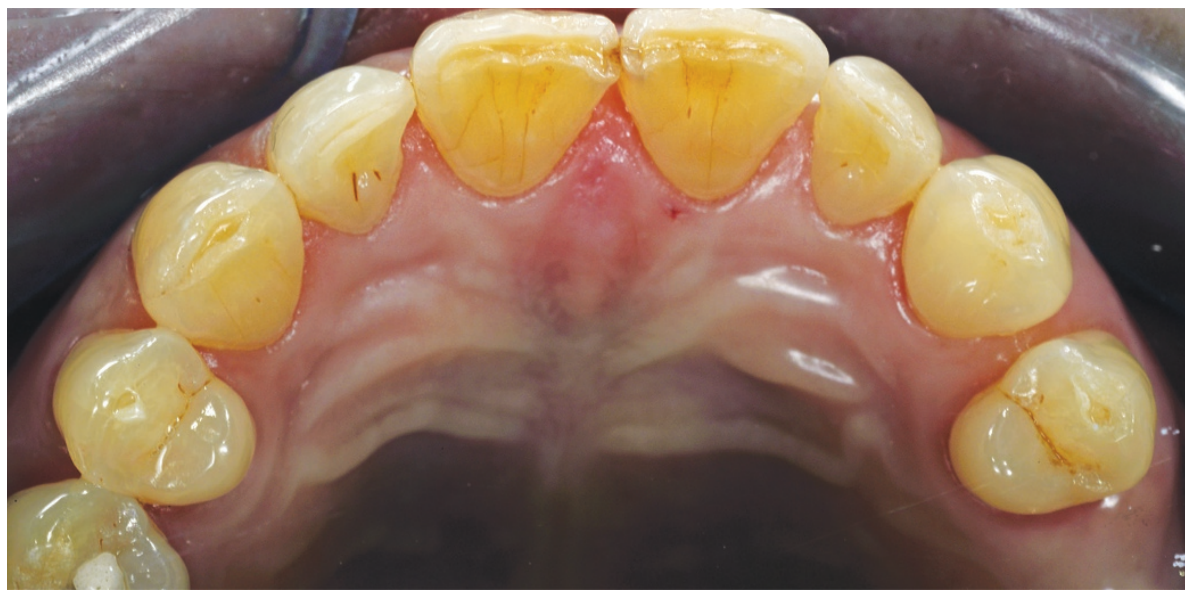

Fig. 8 Final image after cropping, correcting exposure, orientation and laterally inverting (compare with Fig. 3)

\section{FILE FORMATS}

An original in digital photography is only possible at the operating level system, ie within the initial proprietary software that captured the image. Once the image data is opened and subsequently saved in another software package such as photo-editing, graphics, desk top publishing or presentation applications, the original data is altered and irretrievably lost. Alterations include change of colour space, reduction of colour depth or dynamic range. Although the deterioration is negligible and rarely perceptible on a computer monitor, vast numbers of manipulations severely affect image quality if a section of the image is enlarged. Therefore, before opening the image in another type of software, the original should be stored for subsequent retrieval. Furthermore, the way in which the data is archived is essential to reduce alterations, including choosing an appropriate file format. At present there is no file format that is suitable for all circumstances and therefore several types are needed depending on the intended use of the image.

The basic difference between formats is whether the data is compressed or noncompressed, and if compression is applied, whether it is lossless or lossy. The choice of file format is as perplexing as choosing a digital camera. Some examples of image file formats are RAW, PSD, GIF, TIFF, JPEG, PNG, EPS, LZW, DCS, EPS, PICT, Bitmap, etc (Fig. 9). In addition, each camera manufacturer and software developer has their own philosophies regarding the type of image file that best serves digital image data. As yet there is no industry standard, and this adds to the confusion during decision-making. However, to simplify matters, most DSLRs offer the option of selecting three file formats: RAW data, TIFF and JPEG (various varieties with different quality levels) (Fig. 10). Table 1 summarises their salient features and differences.

\section{Proprietary RAW data}

Many camera manufacturers have developed their own file formats to capture the initial image as raw data. These files are camera specific and cannot be opened in any other software except that provided by the camera company. The objective is to capture as pure a digital signal as possible before being processed in the 
capture software and exported into a format that is recognisable by popular manipulation software.

\section{TIFF (Tagged Image File Format)}

If there is an industry standard for image file format, then TIFF is a strong contender. Invented by the Aldus Corporation, TIFF is as 'generic' as an image format can be, and can be opened in nearly all types of manipulation software for data exchange, for example Adobe $^{\varpi}$ Photoshop, Adobe ${ }^{\varpi}$ InDesign, PageMaker ${ }^{\mathrm{TM}}$ or Quark Xpress ${ }^{\mathrm{TM}}$. The most endearing feature of this format is that the compression is lossless in the LZW mode, named after its designers Lempel-Ziv and Welch, and hence there is no detail loss from the raw proprietary precursor file. TIFF can be selected on camera menus as the choice for capturing an image. The advantage is that the camera software performs the necessary white balance and other calibrations to the image, which is ready to be opened in any software of choice. However, to be absolutely pedantic the data is not as pure as the proprietary raw format since the incamera software is rarely as sophisticated as the proprietary capture software. The files generated in the TIFF mode are large, ranging from a few megabytes to over $200 \mathrm{MB}$, depending on camera specifications. Consequently, large capacity storage media are an essential requirement.

\section{JPEG (Joint Photographic Experts Group)}

Unlike TIFF, when JPEG files are opened and saved they suffer from severe lossy compression, with loss of detail especially of diagonal lines in an image (Fig. 11). Various levels of JPEG compression are possible, ranging from Level 10 to Level 1. The higher the level, the larger and higher the quality of the file. A newer version is available called JPEG 2000 with less deterioration in image quality than its predecessor. JPEG files can be as small as a few kilobytes to several megabytes depending on the level of compression. Because of their smaller size, they are ideal for Internet use, particularly for email attachments for communication between members of the dental fraternity. Some cameras also offer the facility to choose JPEG as the initial captured image. The temptation is that the file is small so more pictures can be taken

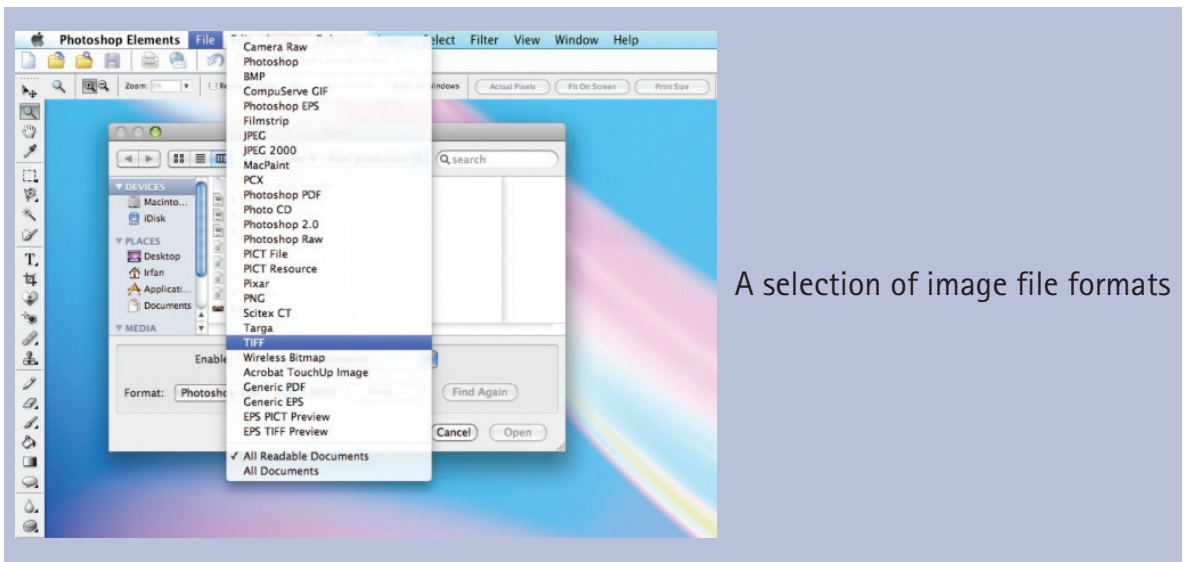

Fig. 9 A selection of image file formats

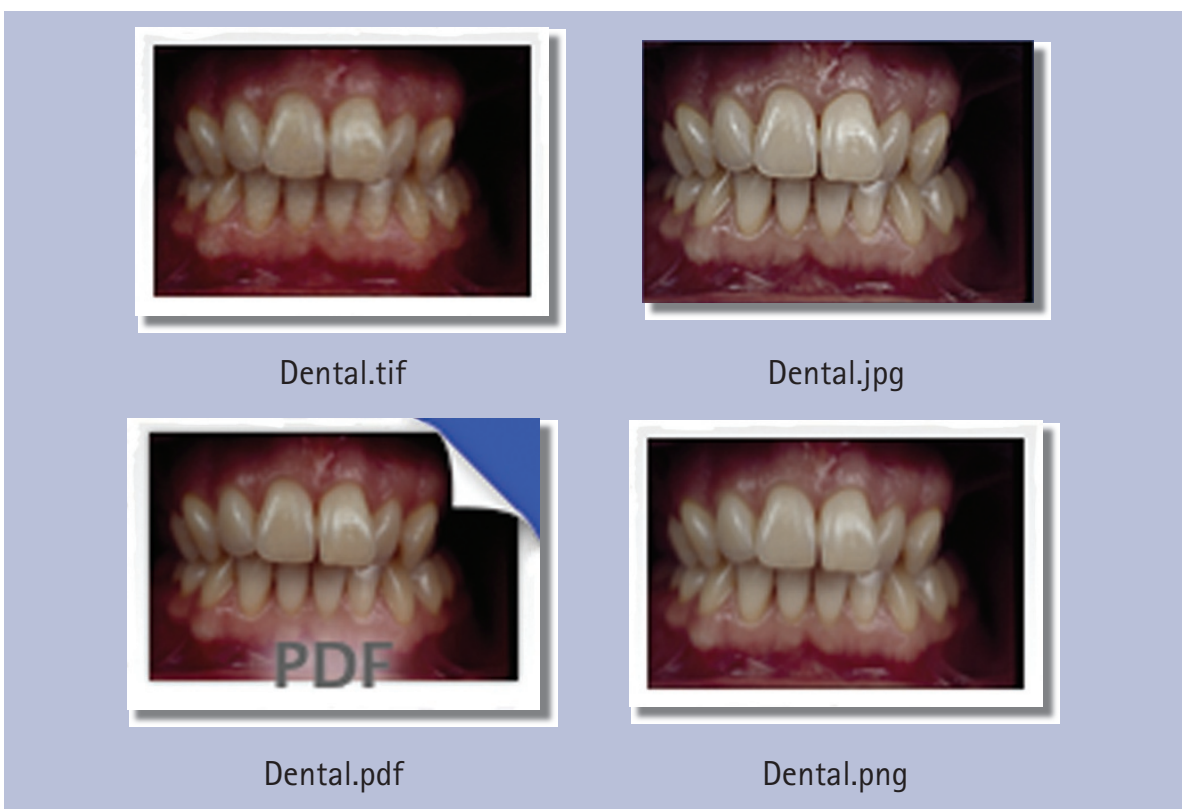

Fig. 10 Icons of popular file formats, TIFF, JPEG 2000, PDF and PNG 24

\begin{tabular}{|c|c|c|c|}
\hline & RAW data & TIFF & JPEG (varieties) \\
\hline Colour mode & RGB & RGB, CMKY, Lab & RGB, CMYK \\
\hline Colour depth/channel & Up to $16 \mathrm{bit} / \mathrm{channel}$ & Up to $16 \mathrm{bit} / \mathrm{channel}$ & 8 bit/channel \\
\hline ICC-profile & - & Yes & Yes \\
\hline Compression & No & Optional & Lossy \\
\hline Alpha channels & No & Yes & No \\
\hline Web-suitable & No & No & Yes \\
\hline
\end{tabular}

before the memory card is full, and the image is automatically calibrated for white balance, etc. However, the temptation should be resisted because the resulting image is of poor quality and unsuitable for archiving. Furthermore, it is better practice to capture the initial image as either a raw or TIFF file which is subsequently easily converted to a JPEG for dissemination.
Unfortunately, conversion in the reverse direction, that is, JPEG to TIFF will not regain the lost image quality.

\section{PDF (Personal Document Files)}

Similar to JPEG files, PDF files are lossy compressed files that are relatively small in size and therefore ideal for electronic transmission. In addition, PDF files also 
have different levels of quality, depending on the chosen parameters before the file is exported. The advantage of PDF files is that they can contain layouts with text, vector drawings and photographic images for distribution via the Internet. These files are very helpful for assessing layout for publishing and can also be used for communication with a dental technician by writing text over images, for example marking parts of a restoration that requires adjustment at the try-in stage. Most graphic software allows files to be exported to a PDF format, and the email recipient can view the files by installing Adobe Acrobat, which is freely available as a download from the Internet.

\section{PNG (Portable Network Graphics)}

PNG file format is a development of the GIF (Graphic Interchangeable Format) file, intended primarily for use on the Internet and for building websites. The GIF format has its origins from the very beginnings of the Internet, but has the major drawback of yielding poor image quality. On the other hand, PNG files have addressed the quality issue as well as delivering faster Interest access. The main reason for this is that PNGs can support up to 24 bit (PGN 24) data, and are therefore capable of retaining quality for developing web pages.

\section{EPS (Encapsulated PostScript)}

EPS files are worth mentioning because they are primarily used for pre-press stages of the printing process (to be discussed further in Part 10). These are vector-orientated files (text and drawings) but can also store pixel-based images with lossless compression. Therefore, this format is ideal for publishing that combines text and images, such as practice stationery, brochures and leaflets. After designing the layout in a graphics application, the file is converted into EPS, ready for transmission to a printing house or an office laser printer that supports the Adobe PostScript $^{\text {TM }}$ printer language.

\section{IMAGE STORAGE AND TRANSFER}

The final stage of processing is to store and transfer the image for safekeeping and intended use, respectively. As previously mentioned throughout this series, dental

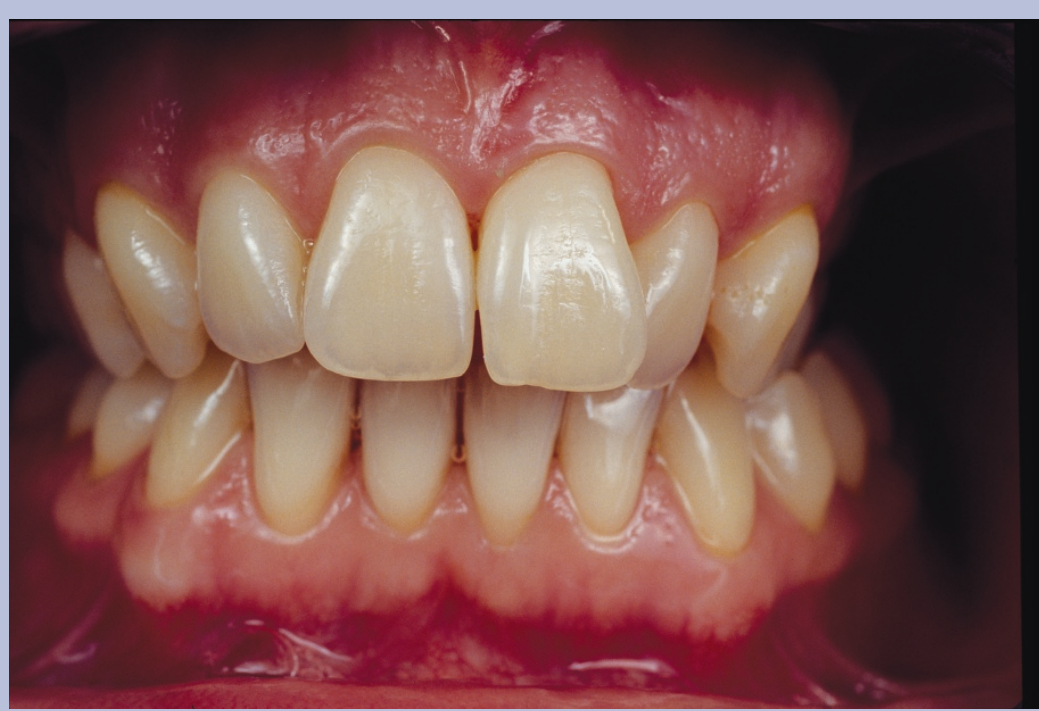

TIFF file format retains image quality (size $=30.9 \mathrm{MB}$ )

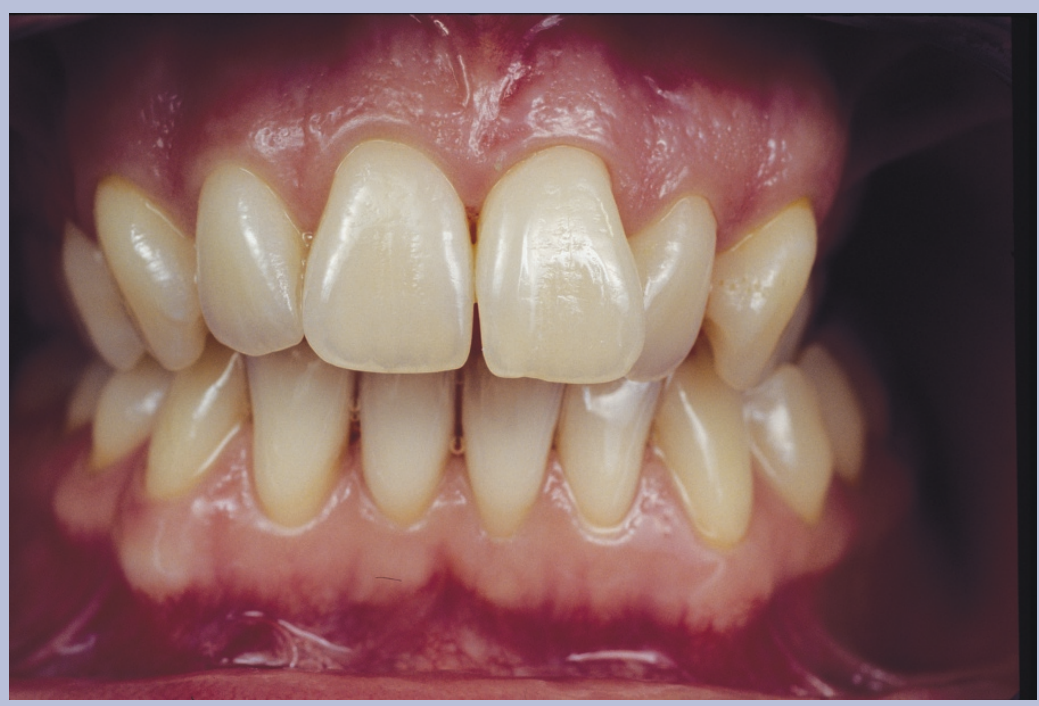

PNG 24 file format retains image quality for building websites (size $=9.1 \mathrm{MB}$ )

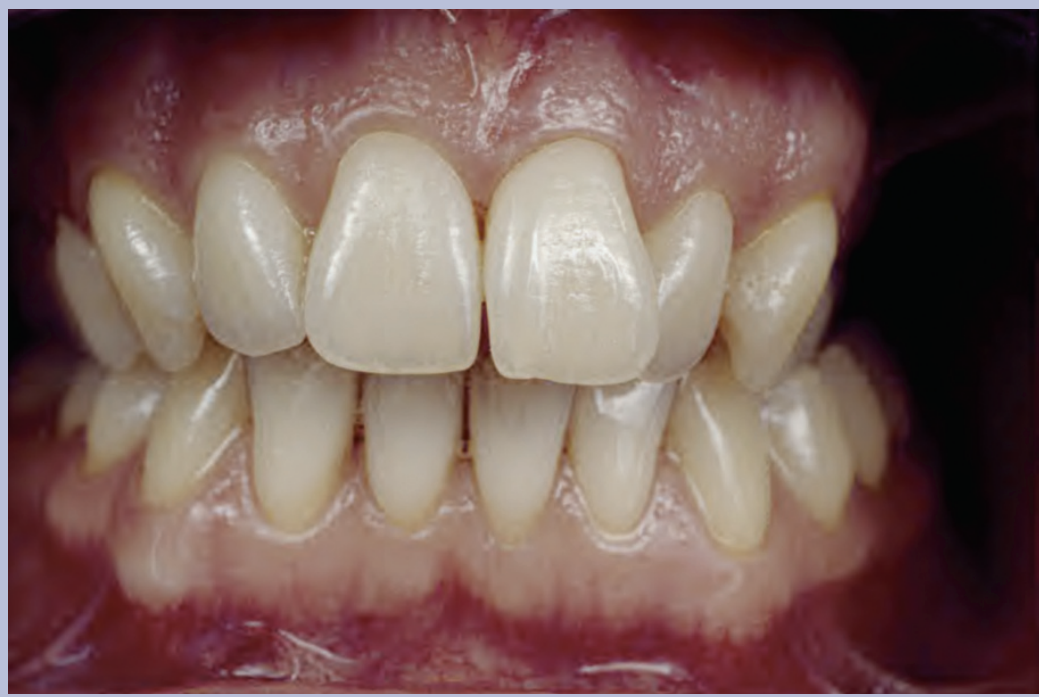

JPEG file format results in deterioration of image quality (size $=812 \mathrm{~KB}$ )

Fig. 11 Comparison of an image saved as TIFF, PGN 24 and JPEG, with severe deterioration in image quality in the latter format 


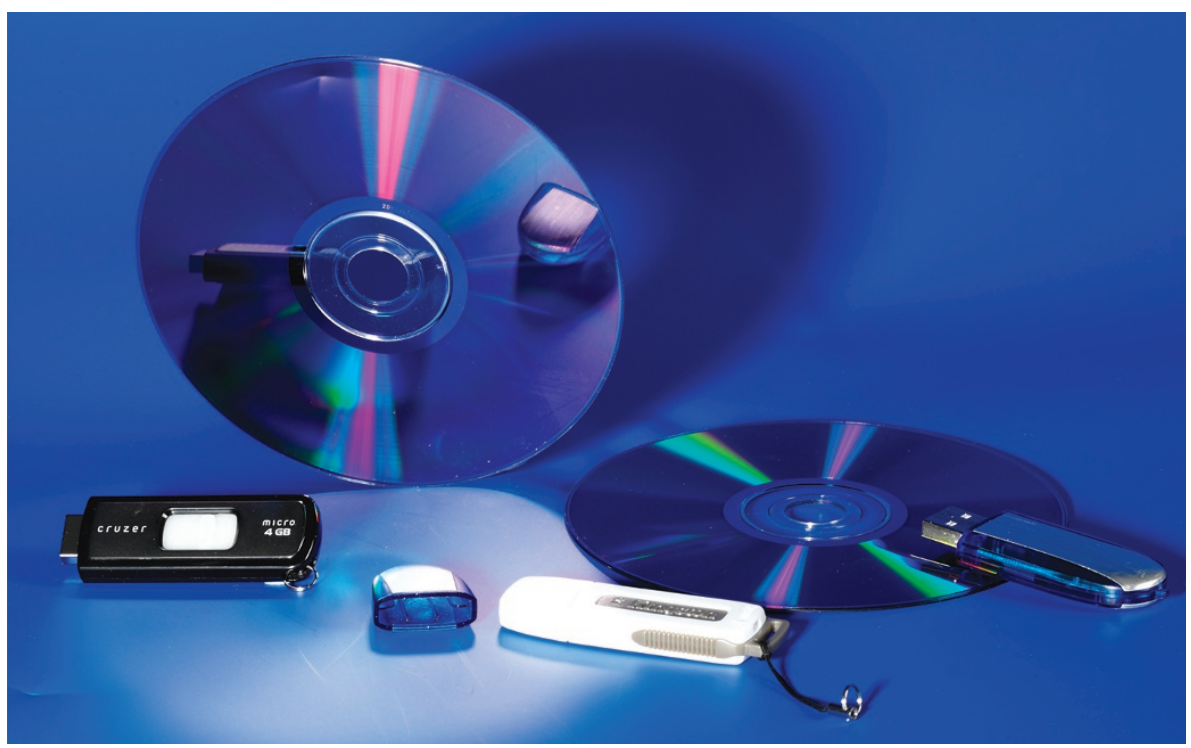

Fig. 12 A selection of portable storage media

\begin{tabular}{|c|c|c|}
\hline Intended use & File & Method of transfer \\
\hline Communication between colleagues & JPEG & Email attachments \\
\hline Layout approval for stationery, brochures & PDF & Email attachments \\
\hline Web publishing & PGN, JPEG & Internet, $C D, D V D$, flash drives \\
\hline Print publishing & TIFF, EPS & $\begin{array}{l}C D, D V D \text {, flash drives or high speed } \\
\text { Internet transfer }\end{array}$ \\
\hline Presentations & High quality JPEG, TIFF & $\begin{array}{l}\text { Via DVI or VGA ports from computer } \\
\text { to projector }\end{array}$ \\
\hline Laser or inkjet office printing & $\begin{array}{l}\text { High quality JPEG, high } \\
\text { quality PDF, TIFF, EPS }\end{array}$ & $\begin{array}{l}\text { USB or wireless connection to } \\
\text { printer }\end{array}$ \\
\hline
\end{tabular}

images are sensitive data and stringent practice protocols must be in place for their storage and transfer to prevent inadvertent loss. Before an image is transferred, the original file must first be securely archived for future retrieval. The best format to store the original is in an unadulterated manner, either in a raw data or a TIFF file format. The following storage protocols are advisable:
1. Create a folder with the patient's name

2. Within the folder, create sub-folders according to when the series of images were taken, for example 'Preoperative status', 'Oral lesions', 'Tooth preparation', 'Temporisation', etc

3. Name each file with a unique name, for example date of image and specific views such as facial, dentofacial, occlusal, etc

4. In addition to a unique name, also add if the file is simply RGB or processed RGB (ie with exposure and orientation correction, etc) before archiving.

Many types of image data management and database software are available which expedite the above procedures, making retrieval easier and more efficient.

High quality images demand huge hardware storage capabilities. As well as fixed storage devices such as computer hard drives, many portable options are available such as CD, DVD, flash sticks and memory cards (Fig. 12). Each of these media should be stored in different locations and if necessary, periodically updated and checked to verify the stored data.

Before an image file is transferred, the intended use must be defined. The use of the picture will determine the type of export file required and the physical method of transfer. Table 2 summarises the intended uses of the images and the ideal file formats and modes of transfer.

1. Ahmad I. Digital dental photography. Part 6: camera settings. Br Dent J 2009; 207: 63-69. 